\title{
Tricuspid regurgitation in the diagnosis of chromosomal anomalies in the fetus at 11-14 weeks of gestation
}

\author{
I C Huggon, D B DeFigueiredo, L D Allan
}

Heart 2003;89:1071-1073

See end of article for authors' affiliations

......................

Correspondence to:

Professor Lindsey D Allan,

Fetal Cardiology Unit,

Harris Birthright Research

Centre for Fetal Medicine,

King's College Hospital,

Denmark Hill, London

SE5 9RS, UK ;

la48@columbia.edu

Accepted 20 ctober 2002

\begin{abstract}
O bjective: To a nalyse patient data to elucidate the apparent association between an abnormal karyotype and tricuspid regurgitation found during fetal echocardiography at early gestations.

Setting: Tertiary referral centre for fetal medicine and cardiology.

Methods: Fetuses between 11 and 14 weeks' gestation were selected for detailed echocardiography. Referral reasons were increased nuchal translucency, a suspected cardiac or extracardiac malformation, and a family history of cardiac malformation.

Intervention: The fetus was imaged transabdominally. The four chamber view, outflow tracts, arterial duct, and aortic arch were assessed on cross sectional imaging and colour flow mapping. Pulsed Doppler of the atrioventricular valves was recorded if possible. Subsequently, the fetal karyotype was ascertained by chorionic villous sampling.

Results: Pulsed Doppler recording of the tricuspid valve was obtained for 262 fetuses. Tricuspid regurgitation was present in $70(27 \%)$ of these, of whom 58 (83\%) proved to have karyotype anomalies. In contrast, $68(35 \%)$ of those without tricuspid regurgitation were found to have karyotype anomalies (95\% confidence interval $36 \%$ to $59 \%, p<0.001$ ). Structural heart defects were detected in 34 of the $58(59 \%)$ with tricuspid regurgitation and in $22(32 \%)$ of those without. The chromosome defect most frequently found to be associated with tricuspid regurgitation was trisomy 21 , but all types of karyotypic anomalies were seen in association.

Conclusion: A careful search for tricuspid regurgitation is an important aspect of the evaluation of the early fetus, as this is frequently a marker for chromosomal defects even in the absence of structural heart disease.
\end{abstract}

ncreased nuchal translucency between 11 and 14 weeks of gestation has been identified as a marker for chromosomal anomalies and for isolated congenital heart disease (CHD). ${ }^{12}$ As our department acts as a referral centre for nuchal measurement, a large selected population is seen who are at high risk for CHD. In a previous paper, ${ }^{3}$ we described the feasibility and accuracy of fetal heart scanning at this early gestation. Fetuses with a nuchal measurement over $4 \mathrm{~mm}$ and those in whom a fetal malformation is suspected routinely have echocardiography immediately before chorionic villous sampling. During this evaluation, it was observed that tricuspid regurgitation was sometimes detected on pulsed Doppler. This finding frequently appeared to predict an abnormal karyotype, even in the absence of a structural cardiac malformation. The object of our present study was to assess the veracity of this suspicion.

\section{METHODS}

Fetuses were examined transabdominally between 11 weeks and 13 weeks 6 days of gestation using an Acuson Aspen system (M ountain View, California, USA) equipped with a $7 \mathrm{mHz}$ curvilinear transducer. All fetuses were examined by cross sectional imaging and colour flow mapping. There was reliable documentation of the pulsed Doppler flow profile in the tricuspid valve and a known karyotype in 262 fetuses seen between April 2000 and December 2001. The indication for echocardiography in these fetuses was increased nuchal translucency in 227, suspected cardiac or extracardiac abnormality in 15, a family history of CHD in four, risk factor in a co-twin in five, abnormal flow in the ductus venosus in nine, and others in two. The presence or absence of tricuspid regurgitation was related to the subsequent karyotype result for each fetus and to the outcome.
Statistical analysis

The $95 \%$ confidence interval was calculated for the difference in the proportions with chromosomal abnormalities of those with and those without tricuspid regurgitation. ${ }^{4}$ The same data were also analysed using the $\chi^{2}$ test taking a threshold of $p<0.001$ as significant.

\section{RESULTS}

Tricuspid regurgitation (or right atrioventricular val ve regurgitation in the setting of an atrioventricular septal defect) was detected in 70 (26.7\%) of the 262 fetuses. Tricuspid regurgitation, when detected, was seen on pulsed flow Doppler with the sample volume positioned in the right atrium at the tricuspid orifice. When a good tracing was obtained, the regurgitation was usually recorded through most of systole and was repeatable and consistent on repeated sampling (fig 1). However, even when recorded on pulsed Doppler, tricuspid regurgitation was often not evident on colour flow mapping, despite the use of colour flow mapping in every case.

Of the 70 fetuses with tricuspid regurgitation, 58 (83\%) had an abnormal karyotype. In contrast, of the 192 fetuses without tricuspid regurgitation, 68 (35\%) had karyotype abnormalities. The 95\% confidence interval for the difference between these proportions was $36.3 \%$ to $58.8 \%$, indicating a significant and substantial difference $\left(\chi^{2}=46.3, p<0.001\right)$. Of the 70 fetuses with tricuspid regurgitation, 40 (57\%) had structural heart disease, of whom 34 (85\%) had an abnormal karyotype. Figure 2 summarises these results.

In 195 fetuses there was no evidence of a structural heart defect. Of these tricuspid regurgitation was detected in 30, 24 $(80 \%)$ of whom had chromosomal anomalies. In the 165 fetuses without tricuspid regurgitation, chromosomal anomalies were present in 46 ( $28 \%$ ) (95\% confidence interval for difference in proportions $36.3 \%$ to $68.0 \%, \chi^{2}=30.0, p<0.001$ ). 


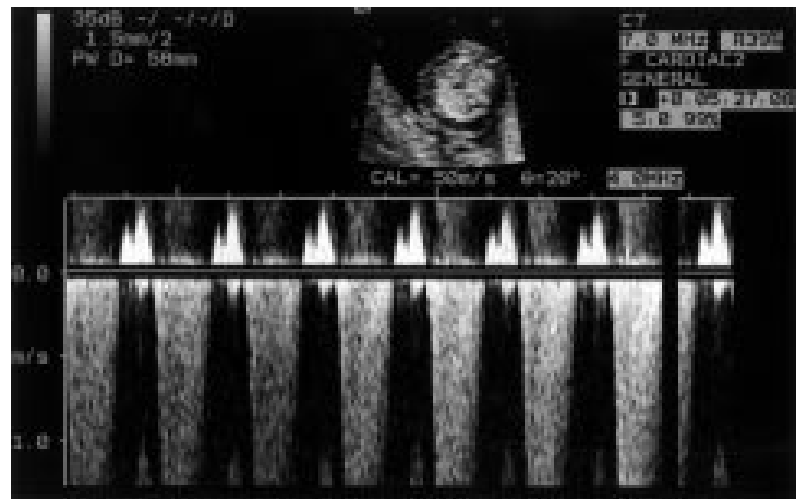

Figure 1 Holosystolic regurgitation is seen on pulsed Doppler examination of the tricuspid valve in a fetus at 12 weeks' gestation. The velocity of over $1 \mathrm{~m} / \mathrm{s}$ allows clear distinction from arterial flow, which is usually less than $50 \mathrm{~cm} / \mathrm{s}$ at this gestational age.

Table 1 details the frequency of tricuspid regurgitation and of structural heart defects identified in the 126 fetuses with chromosomal abnormality of different types. As well as being the most common chromosomal abnormality, trisomy 21 was also the chromosomal abnormality most likely to be associated with tricuspid regurgitation. However, the incidence of structural heart defects diagnosed in trisomy 21 was less than that for the other chromosomal abnormalities.

Outcome data were available for 254 of 262 pregnancies. In 137 cases, the pregnancy was terminated because of the presence of a karyotype abnormality, repeated evidence of structural anomaly, or progressive hydrops. There were eight spontaneous intrauterine deaths and one neonatal death. There were 74 normal live births and a further 34 known continuing pregnancies at the time of a normal later scan at 20 weeks' gestation. Eight pregnancies were lost to follow up. Of note, tricuspid regurgitation was found only in those fetuses referred for increased nuchal translucency or for a suspected fetal anomaly. It was not found in any of the 20 fetuses referred for other causes, all of whom had a normal outcome.

\section{DISCUSSION}

Eighty seven per cent of our cases were referred because of increased nuchal translucency, most of which were over $4 \mathrm{~mm}$, representing a population at very high risk of chromosomal anomalies. This is shown by our results, with $48 \%$ of the fetuses scanned proving to have chromosomal anomalies.
Table 1 Association of chromosomal anomalies with tricuspid regurgitation and CHD

\begin{tabular}{lcll}
\hline Diagnosis & $\begin{array}{c}\text { Number } \\
\text { of cases }\end{array}$ & $\begin{array}{l}\text { With trisuspid } \\
\text { regurgitation }\end{array}$ & With CHD \\
\hline Trisomy 21 & 64 & $38(59 \%)$ & $20(31 \%)$ \\
Trisomy 18 & 27 & $10(33 \%)$ & $13(48 \%)$ \\
Trisomy 13 & 12 & $6(45 \%)$ & $7(58 \%)$ \\
Turner's syndrome & 15 & $3(23 \%)$ & $9(60 \%)$ \\
O thers & 8 & $1(12.5 \%)$ & $5(63 \%)$ \\
Total & 126 & 58 & $54(43 \%)$ \\
\hline CHD, congenital heart disease. & & \\
\hline
\end{tabular}

Tricuspid regurgitation is commonly seen during echocardiography in children either on colour flow mapping or by pulsed Doppler and is found in up to $70 \%$ of normal studies. ${ }^{5}$ However, it is not a common feature seen during fetal echocardiography in the 18-40 week gestational time frame, either on pulsed Doppler or on colour flow mapping. ${ }^{67}$ When tricuspid regurgitation is seen in an otherwise normal fetal heart, it tends to be transitory and of no importance. ${ }^{8}$ Tricuspid regurgitation was not detected during pulsed Doppler examination of atrioventricular valves in the normal early fetal heart in a study of 48 fetuses by Leiva and colleagues. ${ }^{9}$ Thus, the frequency of this finding in our series, occurring in $27 \%$ of cases, was surprising. Caution is advised in extrapolating the results of our study, obtained in a very high risk population, to low risk fetuses. However, the apparent absence of tricuspid regurgitation in low risk fetuses in the study of Leiva and colleagues $^{9}$ and in the few fetuses with normal nuchal translucency in this study suggests that its occurrence, even in low risk fetuses, should not be dismissed.

The sensitivity of colour flow mapping to detect tricuspid regurgitation in the small fetal heart varies considerably depending on the settings and the particular machine used. We attribute our inability to detect tricuspid regurgitation on colour flow mapping on some occasions, when it was readily detected on pulsed wave Doppler, to the colour Doppler limitations of our particular machine, rather than to the trivial nature of tricuspid regurgitation. Our point here is that colour cannot be relied on at this early stage of gestation to exclude regurgitation and that the atrioventricular valves should be individually sampled by pulsed Doppler, which is a more consistent modality between different machines.

This analysis confirms our suspicion that tricuspid regurgitation has a poor prognostic implication, as its presence increased the likelihood of the karyotype proving to be abnormal in a given case. Chromosomal anomalies were detected in

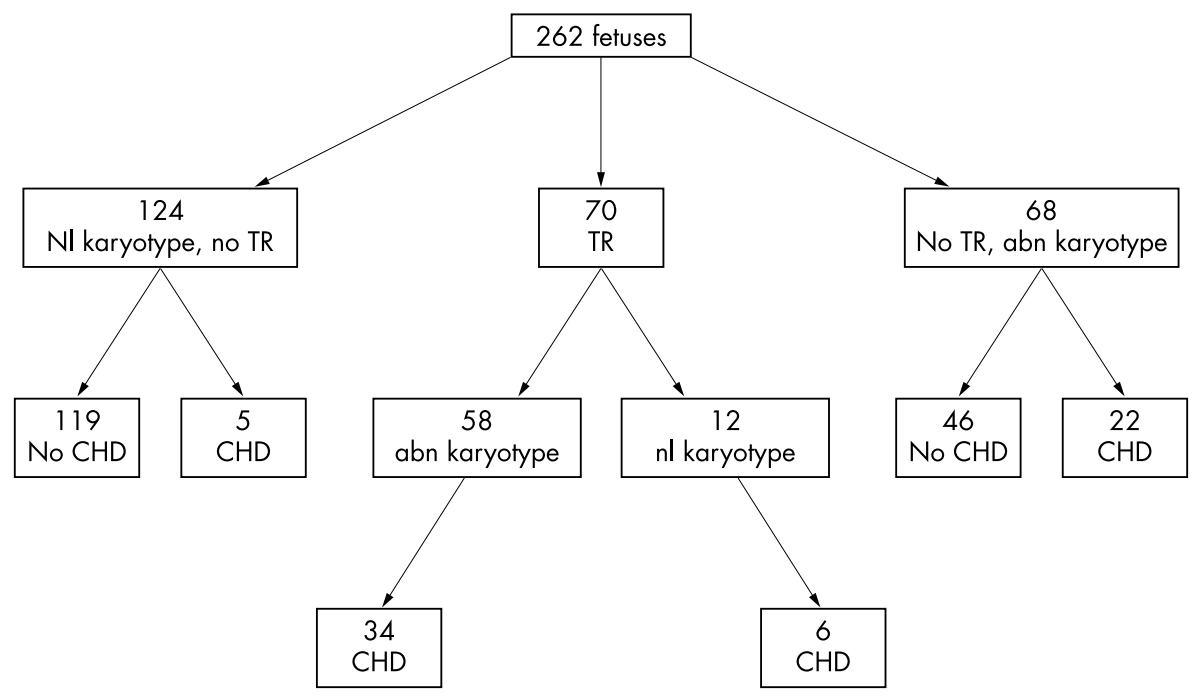

Figure 2 Breakdown of 262 fetuses scanned between 11 and 13 weeks 6 days of gestation. abn, abnormal; CHD, congenital heart disease; $\mathrm{nl}$, normal; TR, tricuspid regurgitation. 
$83 \%$ of those with tricuspid regurgitation but in only $35 \%$ of those without. Structural heart disease was detected in 54 (43\%) of those fetuses who had a chromosome anomaly. Tricuspid regurgitation occurred in 58 (46\%) of the fetuses with a chromosome anomaly. Some types of heart defect associated with chromosomal abnormality such as atrioventricular septal defect may be expected to predispose to atrioventricular valve regurgitation. However, the association of tricuspid regurgitation with chromosomal abnormality did not appear to be accounted for by the association of detectable heart defects with chromosomal anomalies. Even when all fetuses with detectable structural heart defects were excluded from the analysis, there remained a strong association between tricuspid regurgitation and chromosomal anomalies. Therefore, tricuspid regurgitation occurred in the absence of detectable heart disease in 24 cases, and the majority of those fetuses ( 18 of 24) proved to have trisomy 21 . It may be argued that a cardiac anomaly was present in those fetuses as an explanation for tricuspid regurgitation, but we were unable to detect it on this early study. Certainly, one fetus in our previous series ${ }^{3}$ proved to have an atrioventricular septal defect at necropsy, which had not been suspected on scan. On the other hand, in that series the diagnosis of either a normal heart or a cardiac malformation was confirmed at postmortem examination in 26 of 28 cases. Thus, it seems unlikely that many cases of major cardiac malformation were overlooked.

In this current series, two of the authors reviewed the videotapes independently and only those cases where there was agreement on the diagnosis, and on there being adequate quality of images for diagnosis, were included. It is of interest that although $60 \%$ of fetuses with Turner's syndrome had a cardiac malformation, relatively few (20\%) had tricuspid regurgitation. In contrast, only a third of the fetuses with trisomy 21 had a cardiac malformation but 59\% had tricuspid regurgitation. It is of note that tricuspid regurgitation has also been seen in the trisomic mouse fetus, occurring in about $25 \%$ of those examined at similar gestational stages to the 12 week human fetus. ${ }^{10}$ Although not widely accepted and not our experience, a link between atrioventricular valve regurgitation and trisomy 21 has been previously noted in the 16-22 weeks' gestational age range. ${ }^{11} 12$

The aetiology of the tricuspid regurgitation is questionable. It may be caused by an increase in preload, or volume, presented to the right ventricle. Against this is the lack of cardiac or right ventricular dilatation, which may be expected if there were volume overload. Alternatively, the right ventricle may face an increase in afterload. The afterload of both ventricles is normally the same in the fetus in the absence of an aortic arch anomaly. However, mitral regurgitation was found in only one fetus in this series, perhaps because its cardiac structure was more anatomically suited to withstanding a pressure or volume load. The afterload of both ventricles is dominated by the placental resistance, which is high in early pregnancy and falls around the 12-16 week period. This is consistent with the fact that tricuspid regurgitation was not found in five of seven fetuses who had it when they were examined later in pregnancy. This may have a physiological link with increased nuchal translucency, which is known to be usually a transitory finding in the normal fetus and in the fetus with CHD or a chromosomal anomaly.

\section{Study limitations}

We recognise the limitations of this study, which is partly retrospective. The implication of finding tricuspid regurgitation was not appreciated early in our experience and the search in every fetus examined before J une 2001 to detect or exclude this finding was perhaps not sufficiently careful. Additionally, although the smallest sample volume is large relative to the size of the fetal heart at 12 weeks of gestation, it must be positioned in the right atrium at the orifice of the tricuspid valve to detect regurgitation. If it is positioned wholly in the right ventricle, a normal tricuspid flow profile would be obtained, but without regurgitation evident even when it is present. Thus, some cases of tricuspid regurgitation in this series may have been overlooked. On the other hand, although the ability to analyse the heart is largely dependent on the fetal position, skill in this difficult early scanning has improved over time and this may have increased the detection of tricuspid regurgitation latterly.

\section{Conclusion}

Significant, substantial tricuspid regurgitation is frequently detected by pulsed Doppler during early cardiac assessment of a population of fetuses at high risk for chromosomal and other anomalies. It is commonly a marker for cardiac malformations, but even in the absence of detectable structural heart disease it is highly associated with chromosomal anomalies, particularly trisomy 21.

\section{ACKNOWLEDGEMENTS}

LDA is supported by the Fetal M edicine Foundation.

\section{Authors' affiliations}

I C Huggon, D B DeFigueiredo, L D Allan, Fetal Cardiology Unit, Harris Birthright Research Centre for Fetal Medicine, King's College Hospital, London, UK

\section{REFEREN CES}

1 Nicolaides $\mathrm{KH}, \mathrm{Azar} \mathrm{G}$, Byrne D, et al. Fetal nuchal translucency: ultrasound screening for chromosomal defects in first trimester of pregnancy. BMJ 1992;304:867-9.

2 HyettJ, Perdu M, Sharland GK, et al. Increased nuchal translucency at 10-14 weeks of gestation as a marker for major cardiac defects. Ultra sound O bstet G ynecol 1997:10:242-6.

3 Huggon IC, G hi T, Cook AC, et al. Fetal cardiac abnormalities identified prior to 11-14 weeks gestation. Ultrasound $O$ bstet $G$ ynecol 2002;20:22-9

4 Yoshida K, Yoshikawa J, Shakudo M, et al. Color Doppler evaluation of valvular regurgitation in normal subjects. Circulation 1988:78:840-7.

5 Smrcek JM, G embruch U. Longitudinal observations in normally grow $\mathrm{n}$ fetuses with tricuspid valve regurgitation: report of 22 cases. Prenat Diagn 1999;19:197-204

6 Respondek ML, Kammermeier M, Ludomirsky A, et al. The prevalence and clinical significance of fetal tricuspid valve regurgitation with normal heart a natomy. Am J O bstet G ynecol 1994;171:1265-70.

7 Gembruch U, Smrcek JM. The prevalence and clinical significance of tricuspid valve regurgitation in normally grown fetuses and those with intrauterine grow th retardation. Ultrasound 0 bstet $G$ ynecol $1997 \cdot 9 \cdot 374-82$

8 Gardner MJ, Altman DG, eds. Statistics with confidence. London: British Medical Journal,1989:28-33.

9 Leiva MC, Tolosa JE, Binotto CN, et al. Fetal cardiac development and hemodynamics in the first trimester. Ultrasound $O$ bstet $G$ ynecol $1999 ; 14: 169-74$

10 Gui Y, Linask KK, Khow sathit $P$, et al. Doppler echocardiography of normal and abnormal embryonic mouse heart. Pediatr Res 1996:40:633-42.

11 DeVore GR. Trisomy 21: $91 \%$ detection rate using second-trimester ultrasound markers. Ultrasound 0 bstet $G$ ynecol 2000;16:133-41.

12 DeVore GR, Alfi 0 . The use of color Doppler ultrasound to identify fetuses at increased risk for trisomy 21: an alternative for high-risk patients who decline genetic amniocentesis. 0 bstet $G$ ynecol $1995 ; 85: 378-86$. 